\title{
Role of chromohysteroscopy in detection of endometrial pathology in abnormal uterine bleeding
}

\author{
Aleena Haider ${ }^{1 *}$, Imam Bano ${ }^{1}$, Noor Afshan Sabzposh ${ }^{1}$, Sayeedul Hasan Arif ${ }^{2}$
}

\begin{abstract}
${ }^{1}$ Department of Obstetrics and Gynecology, ${ }^{2}$ Department of Pathology, Jawaharlal Nehru Medical College, Aligarh Muslim University, Aligarh, Uttar Pradesh, India
\end{abstract}

Received: 16 January 2019

Accepted: 06 February 2019

\section{*Correspondence:}

Dr. Aleena Haider,

E-mail: aleenahaider447@gmail.com

Copyright: $\odot$ the author(s), publisher and licensee Medip Academy. This is an open-access article distributed under the terms of the Creative Commons Attribution Non-Commercial License, which permits unrestricted non-commercial use, distribution, and reproduction in any medium, provided the original work is properly cited.

\begin{abstract}
Background: Abnormal uterine bleeding is one of the most common clinical problems encountered in gynaecological practice. Chromohysteroscopy is thought to improve the diagnostic accuracy of hysteroscopy and reach the final diagnosis in AUB and is the subject matter of this study.

Methods: A prospective cross-sectional study was performed on 35 women with AUB, who presented to the Dept. of Obstetrics and Gynecology, JNMCH, Aligarh, over a period of 2 years (2014-2016). After transvaginal sonography, all cases underwent hysteroscopy followed by chromohysteroscopy using 5\% methylene blue dye. Differentially stained pattern of the uterine cavity was observed and hysteroscopic guided biopsy was taken from stained and unstained areas, followed by endometrial aspiration biopsy. All three samples were sent for histopathological examination. Results were analyzed and compared.

Results: Out of 35 cases studied, 13 cases showed diffuse light staining, while 22 cases had focal dark staining. Histopathology results from diffuse light stained group showed 9 (69.2\%) had normal endometrium. Out of 22 cases with focal dark staining, $20(90.0 \%)$ cases had abnormal histopathology. It was found that focal dark staining to detect endometrial pathology had a sensitivity of $83.3 \%$, specificity of $81.8 \%$, positive predictive value of $90.9 \%$, negative predictive value of $69.2 \%$ and accuracy $82.8 \%$. The difference in the diagnostic ability of stained tissue biopsy was highly significant $(\mathrm{p}<0.001)$ when compared to blind endometrial aspiration biopsy.

Conclusions: Chromohysteroscopy is an efficacious simple tool for detecting endometrial pathology in cases of AUB.
\end{abstract}

Keywords: Abnormal uterine bleeding (AUB), Chromohysteroscopy, Endometrial aspiration biopsy, Hysteroscopy

\section{INTRODUCTION}

Abnormal uterine bleeding (AUB) is one of the most common clinical problems encountered in gynecological practice. Up to $30 \%$ of women are affected by heavy menstrual bleeding throughout their reproductive lifetime. ${ }^{1}$ AUB is a broad term used to describe any deviation from normal menstruation or menstrual cycle pattern. $^{2}$ A thorough clinical history and physical examination help to establish the cause of the abnormal bleeding. Imaging techniques including transvaginal sonography allows detailed assessment of anatomical abnormities of uterus and endometrium. ${ }^{3}$ However, endometrial sampling is required to reach the final diagnosis. Traditionally, AUB has been investigated with blind conventional procedures like $\mathrm{D}$ and $\mathrm{C}$, fractional endometrial biopsy etc., but now, diagnostic hysteroscopy with hysteroscopic guided biopsy has become the gold standard diagnostic tool for evaluation of AUB ${ }^{4}$ Although hysteroscopy facilitates adequate visualization of the uterine cavity and detection of intracavitary lesions, a normal appearance of 
endometrium in conventional diagnostic hysteroscopy does not ensure the integrity of endometrial cells. Endometrial pathology in these normal looking areas can be detected by chromohysteroscopy. Chromoendoscopy has been widely used in gastrointestinal imaging to identify mucosal abnormalities by enhancing tissue characterization and differentiation. Based on this technique, Methylene blue was applied to the endometrium during conventional hysteroscopy to detect endometrial pathology that could not be seen by the naked eye during hysteroscopy. Thus, the technique of chromohysteroscopy came into existence. Chromohysteroscopy was introduced after the study by Kucuk and Safali in 2008, in which chromoendoscopy and hysteroscopy was combined to improve the diagnostic value of hysteroscopy in the setting of assisted reproduction. ${ }^{4}$

Chromohysteroscopy, a relatively new procedure, was thought to decrease the subjective error and increase the sensitivity as compared to simple diagnostic hysteroscopy, and thus, was explored for diagnosing AUB in this study. The primary objective of this study was to find out the efficacy of chromohysteroscopy in detection of endometrial pathology in patients of abnormal uterine bleeding. The secondary objective was to compare the histological findings of the stained endometrial tissue with that of unstained endometrium and endometrial aspiration biopsy.

\section{METHODS}

This prospective cross-sectional study was conducted in the department of Obstetrics and Gynaecology, in collaboration with department of Pathology, Jawaharlal Nehru Medical College and Hospital, A.M.U, Aligarh over a period of two years (2014-2016). Ethical clearance was taken from Institutional Ethical Committee. Thirtyfive women presenting with the complaint of abnormal uterine bleeding without any gross identifiable abnormality on clinical examination or investigations, were included in the study after taking informed written consent. A detailed history and clinical examination, followed by investigations including haemogram, LFT, RFT, blood sugar, thyroid profile was done. This was followed by Pap smear and transvaginal sonography. Patients with any identifiable gross abnormality were excluded from the study and managed as per the hospital protocol. Diagnostic hysteroscopy was performed using a $4.2 \mathrm{~mm}$ hysteroscope and all the patients with gross pathology were excluded. In the selected cases, saline infusion was stopped for a while and $10 \mathrm{ml}$ of $5 \%$ Methylene blue dye was instilled through the water inlet channel. After waiting for 5minutes, saline infusion was restarted, and extra dye was washed off. Two types of endometrial staining patterns were observed - diffuse light blue staining and focal dark blue staining. Endometrial biopsies were obtained from both the areas using hysteroscopic grasper forceps (Figure 1). At the end of the procedure, endometrial aspiration was done using Karman's cannula. All the three samples were sent for histopathological examination.

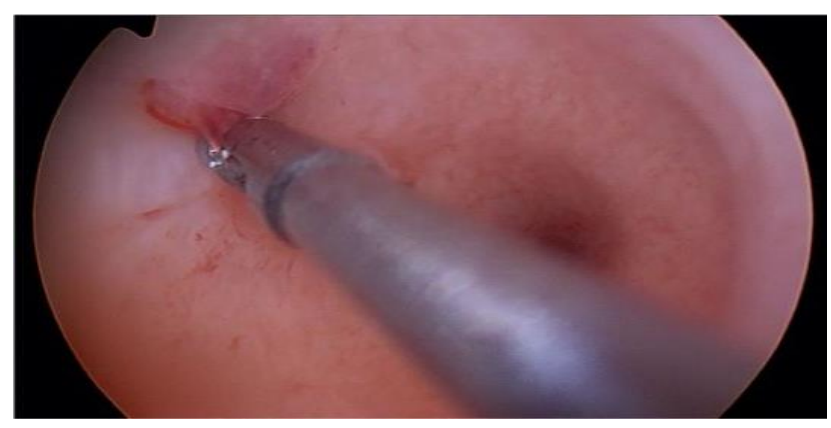

Figure 1: Hysteroscopic guided biopsy of the endometrium with the help of grasper forceps.

Data was recorded and analyzed. $2 \times 2$ table was used to analyze the sensitivity, specificity, positive predictive value and negative predictive value of chromohysteroscopy. Chi square test was used to compare variables between groups. $\mathrm{P}$ value $<0.05$ was considered significant while that, 0.001 was taken as highly significant.

\section{RESULTS}

The mean age of these women was $39.5+/-8.5$ years and most of the cases were in the age group of 35-50 years. Majority of the patients i.e. 21(60\%) out of 35 presented with the symptom of menorrhagia. Only 2 cases $(5.7 \%)$ presented with postmenopausal bleeding.

\section{Pattern of staining}

As shown in Table 1, out of 35 women, 13(37.1\%) had diffuse light staining on chromohysteroscopy (Figure 2) while $22(62.9 \%)$ of them developed focal dark staining (Figure 3).

Table 1: Staining pattern on chromohysteroscopy.

\begin{tabular}{|l|l|l|}
\hline Staining pattern & No. of patients & $\%$ \\
\hline Diffuse light staining & 13 & 37.1 \\
\hline Focal dark staining & 22 & 62.9 \\
\hline Total & 35 & 100 \\
\hline
\end{tabular}

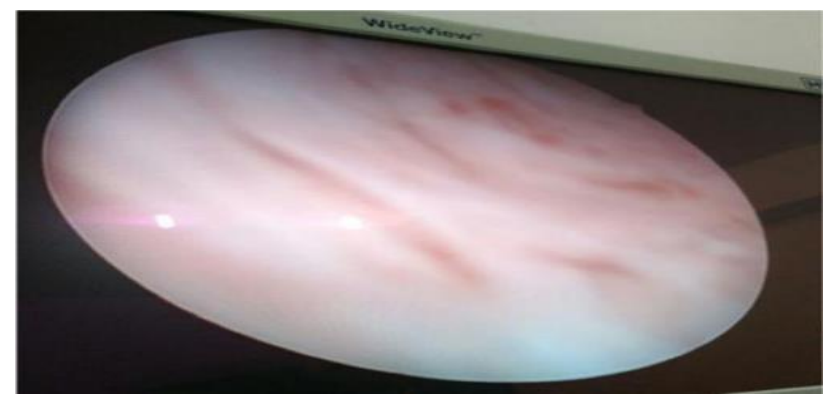

Figure 2: Diffuse light stained endometrium seen on chromohysteroscopy. 


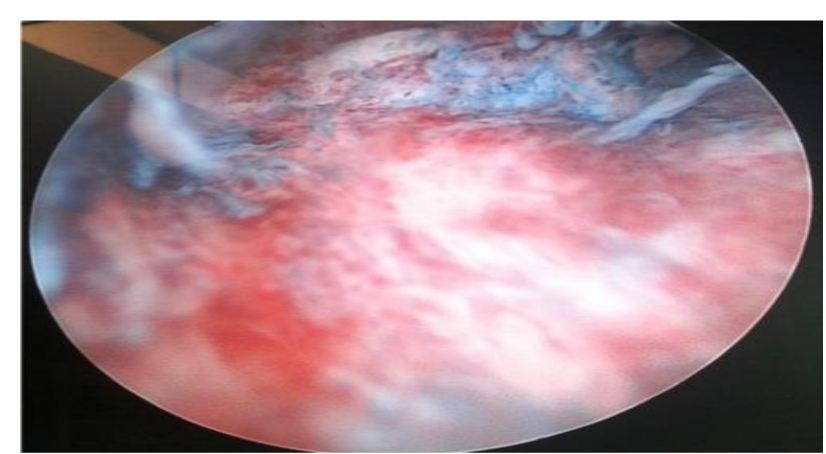

Figure 3: Focal dark staining of damaged endometrium with interspersed unstained areas on staining with methylene blue dye.

\section{Histopathological findings}

As shown in Table 2, among the diffuse light stained group, 9 (69.2\%) patients out of 13, showed normal endometrium on histopathology, while $4(30.8 \%)$ of them had abnormal endometrium, that too in the form of hormonal imbalance. On the other hand, in the focal dark stained group, out of 22 patients, 20 (90.9\%) patients had abnormal endometrium on HPE, while only 2 cases had normal endometrium. Hence, it was seen that patients with focal dark staining on chromohysteroscopy had more abnormal histopathology findings than diffuse light stained group and the difference was found to be highly significant $\left(\chi^{2}=13.7, \mathrm{p}<0.001\right)$.

Table 2: Histopathology according to staining pattern.

\begin{tabular}{|l|l|l|l|}
\hline Stained pattern & $\begin{array}{l}\text { Abnormal } \\
\text { HPE }\end{array}$ & $\begin{array}{l}\text { Normal } \\
\text { HPE }\end{array}$ & $\begin{array}{l}\text { p } \\
\text { value }\end{array}$ \\
\hline \begin{tabular}{l|l|l|} 
Diffuse light \\
stained $(\mathrm{n}=13)$
\end{tabular} & $4(30.8 \%)$ & $9(69.2 \%)$ & \\
\hline $\begin{array}{l}\text { Focal dark stained } \\
(\mathrm{n}=22)\end{array}$ & $20(90.9 \%)$ & $2(9.1 \%)$ & $<0.001$ \\
\hline Total & 24 & 11 & \\
\hline
\end{tabular}

To detect endometrial pathology on chromohysteroscopy, focal dark staining had a sensitivity of $83.3 \%$, specificity of $81.8 \%$, positive predictive value of $90.9 \%$, negative predictive value of $69.2 \%$ and accuracy of $82.8 \%$.

\section{Histopathology results in focal dark stained group}

As seen in Table 3, out of the 22 patients showing focal dark staining, 20(90.9\%) abnormal histopathology reports were obtained from darkly stained area, 14(63.6\%) were detected from unstained area samples, while $13(59.1 \%)$ were obtained from endometrial aspiration biopsy.

Table 3: Histopathology from stained, unstained and aspiration biopsy in focal dark stained group.

\begin{tabular}{|l|l|l|l|l|}
\hline Column1 & HPE report & Stained & Unstained & Aspiration \\
\hline & Proliferative endometrium & 0 & 6 & 3 \\
\hline & Secretory endometrium & 2 & 0 & 6 \\
\hline Percentage & Shedding endometrium & 0 & 36.4 & 40.9 \\
\hline Abnormal & & 9.1 & 2 & 1 \\
\hline & Disordered proliferation & 2 & 2 & 1 \\
\hline & Luteal phase defect & 0 & 0 & 0 \\
\hline & Irregular ripening & 0 & 0 & 1 \\
\hline & TB endometritis & 3 & 4 & 4 \\
\hline & Hyperplasia without atypia & 5 & 0 & 1 \\
\hline & Hyperplasia with atypia & 2 & 1 & 2 \\
\hline & Chronic endometritis & 4 & 1 & 2 \\
\hline & Atrophic endometritis & 3 & 1 & 1 \\
\hline Percentage & Endometrial carcinoma & 1 & 3 & 0 \\
\hline Total & Fibrosis & 0 & 63.6 & 59.1 \\
\hline
\end{tabular}

Maximum cases were of endometrial hyperplasia without atypia; 5 detected by darkly stained samples, and 4 each from unstained area and endometrial aspiration samples. There was one case of endometrial carcinoma, which was detected by all the three methods. Thus, it was seen that stained areas had more abnormal histopathology findings than unstained and endometrial aspiration biopsy.

\section{Comparison of histopathology findings}

As depicted in the Table 4, out of 22 cases which took focal dark staining on chromohysteroscopy, the area which took dark stain revealed abnormal endometrium in $20(90.9 \%)$ cases and normal endometrium in $2(9.1 \%)$ cases. Biopsies taken from unstained areas in these cases 
showed that $14(63.6 \%)$ patients had abnormal histopathology result while $8(36.4 \%)$ of them had normal endometrium. The histopathology from aspiration biopsy of endometrium showed abnormal endometrium in 13 patients $(59.1 \%)$ and $9(40.9 \%)$ cases had normal endometrium. The association of dark staining with abnormal histopathology was found to be statistically significant $[\chi 2=6.7, \mathrm{p}<0.05]$.

Table 4: Comparison of histopathology results in stained, unstained and aspiration biopsy samples.

\begin{tabular}{|l|l|l|l|}
\hline Type of biopsy & $\begin{array}{l}\text { Abnormal } \\
\text { HIPE }\end{array}$ & $\begin{array}{l}\text { Normal } \\
\text { HPE }\end{array}$ & $\begin{array}{c}\text { p } \\
\text { value }\end{array}$ \\
\hline $\begin{array}{l}\text { Stained area } \\
(\mathrm{n}=22)\end{array}$ & $20(90.9 \%)$ & $2(9.1 \%)$ & $<0.05$ \\
\hline $\begin{array}{l}\text { Unstained area } \\
(\mathrm{n}=22)\end{array}$ & $14(63.6 \%)$ & $8(36.4 \%)$ & \\
\hline $\begin{array}{l}\text { Endometrial } \\
\text { aspiration }(\mathrm{n}=22)\end{array}$ & $13(59.1 \%)$ & $9(40.9 \%)$ & \\
\hline
\end{tabular}

\section{DISCUSSION}

The age of patients in present study population varied from 23 years to 54 years with the mean age of 39.5 years which is similar to that in the study, conducted by Singh et al in 60 patients where the mean age was 33.4 years. ${ }^{7}$

In present study, two types of staining patterns were noted- diffuse light staining in $37.1 \%$ patients and focal dark staining in $62.9 \%$ cases (Table 1). Kucuk et al performed chromohysteroscopy for evaluation of endometrium in 63 cases of recurrent IVF failure and observed focal dark staining in 22 cases accounting for almost $35 \% .^{5}$

In present study, diffuse light stained group $(n=13)$ had greater number of patients $(69.2 \%)$ with normal endometrium. On the other hand, in 22 cases of focal dark stained group, only $9.1 \%$ were normal while $90.9 \%$ showed abnormal histopathology report (Table 2). Thus, it could be concluded that the patients with focal dark staining are more likely to have endometrial pathology. This difference was found to be highly significant $(\mathrm{p}<0.001)$. In the present study, focal dark staining could detect endometrial pathology with sensitivity of $83.3 \%$, specificity of $81.8 \%$, positive predictive value of $90.9 \%$, negative predictive value of $69.2 \%$ and accuracy of $82.8 \%$. Similar result was obtained in the study conducted by Mansour et al which showed a significant incidence of chronic endometritis in (7/16) patients with focal dark staining $(43.75 \%)$ in comparison to $(3 / 41)$ patients with diffuse light staining $(7.32 \%){ }^{6}$ They reported that chromohysteroscopy had $70 \%$ sensitivity, $80.8 \%$ specificity, $43.7 \%$ PPV and $92.6 \%$ NPV for diagnosis of chronic endometritis. Kucuk et al performed chromohysteroscopy for evaluation of endometrium in 63 cases of recurrent IVF failure. Focal dark staining was observed in 22 cases. Histopathology showed endometritis in 9 of them..$^{5}$ Thus, chromohysteroscopy had sensitivity of $69.2 \%$, specificity of $74 \%$, positive predictive value of $40.9 \%$ and negative predictive value of $90.2 \%$ for endometritis. Present study has considerably higher sensitivity and specificity but a lower negative predictive value probably because in this study, authors have taken into account all the possible endometrial pathology that can be a cause for abnormal uterine bleeding while the above studies have only considered endometritis.

In the focal dark stained group, when histopathology results of the stained area were compared with that of the unstained, the power of dark staining to detect endometrial pathology was found to be statistically significant $(\mathrm{p}<0.05)$, as shown in Table 4. In a similar study conducted by Singh et al it was found that the diagnostic accuracy of stained tissue biopsy to detect endometrial pathology was higher than that of unstained tissue biopsy and endometrial aspiration with a statistically significant difference $(\mathrm{p}=0.006){ }^{7}$ In another study conducted by El-Faissal et al on a group of 50 postmenopausal women, it was found that chromohysteroscopy for endometrial pathology had a sensitivity of 93.755 , specificity of $27,77 \%$, positive predictive value of $69.76 \%$ and negative predictive value of $71.42 \% .^{8}$ Their study had low specificity as compared to the present study because they did not take into account the varying degrees of depth of staining.

In the present study, only one case of endometrial carcinoma was detected in focal dark staining group, which was positive in all the three biopsy samples, whether taken from stained area, unstained area or endometrial aspiration. In the previous study conducted by Singh et al also, only one case of endometrial carcinoma was found, and it did not show any staining of endometrium. $^{7} \quad$ El-Faissal et al found 4 cases of endometrial cancer in a study population of $50 .{ }^{8}$ Two of these showed focal dark staining and in the other two, whole endometrium was homogenously stained. Therefore, similar to the previous studies, in present study, the role of chromohysteroscopy in distinguishing malignant from nonmalignant tissue could not be evaluated. In present study, none of the patients developed any complication related to the procedure or distention media. Bettochi et al in their study observed no major complications during hysteroscopic procedures. A study by Jansen et al illustrated that for diagnostic procedures, the overall complication rate was $0.28 \% .^{9,10}$

The limitations of the study were its small sample size which led to inadequate statistical analysis. Also, the differentiation in the dark and light stained areas was not objectively graded. Further studies with larger sample size are required to verify its real predictive value.

\section{CONCLUSION}

It is therefore concluded from present study that chromohysteroscopy improves the diagnostic efficacy of 
hysteroscopy in targeting endometrial biopsy. It improves the diagnostic value and provides a method for guided biopsy to diagnose endometrial pathology in the absence of macroscopic abnormalities, showing a definite advantage over blind aspiration biopsy. It is a costeffective technique without any side effects that may be tried in all cases of abnormal uterine bleeding.

Funding: No funding sources

Conflict of interest: None declared

Ethical approval: The study was approved by the Institutional Ethics Committee

\section{REFERENCES}

1. Market Opinion and Research International (MORI). Women's health in 1990. [Research study conducted on behalf of Parke-Davis Laboratories]. London:MORI $; 1990$.

2. Fraser IS, Critchley HO, Broder M, Munro MG. The FIGO recommendations on terminologies and definitions for normal and abnormal uterine bleeding. InSeminars Reproduct Med 2011;29(5):p. 383.

3. Vercellini P, Cortesi I, Oldandi S, Moschetta M, DeGiorgi O, Crosignani PG. The role of transvaginal ultrasonography and outpatient diagnostic hysteroscopy in the evaluation of patients with menorrhagia. Hum Reprod. 1997;12(8):1768-71.

4. OJ Speroff Leon, Fritz MA. Clinical Gynaecologic endocrinology and infertility. 8th ed. Philadelphia: Lippincott Williams and Wilkins; 2011;591-604.
5. Kucuk T, Safali M. Chromohysteroscopy for evaluation of endometrium in recurrent in vitro fertilization failure. J Assist Reprod Genet. 2008;25(23):79-82.

6. Mansoura H, Mohamed A., Value of endometrial dyeing in diagnosis of endometritis in absence of macroscopic abnormalities during hysteroscopy. Middle East Fertil Soc J.2011;16(1):83-6.

7. Singh N, Singh B. Chromohysteroscopy-A new technique for endometrial biopsy in Abnormal Uterine Bleeding (AUB). Open J Obstet Gynecol. 2013;3(05):11.

8. El-Faissal, Yahia M., Ahmed M.Kamel.The Value of Chromohysteroscopy in Assessment of Postmenopausal Vaginal Bleeding. J Clinic Gynecol Obstet. 2014;3(1):35-41.

9. Bettochi S, Selvaggi L. A vaginoscopic approach to reduce pain of office hysteroscopy. J Am Assoc Gynecol Laparosc. 1997;4(2):255-8.

10. Jansen FW, Vredevoogd CB, Van Ulzen K, Hermans J, Trimbos JB, Trimbos, Kemper TC. Complications of hysteroscopy: a prospective, multicenter study. Obstet Gynecol.2000;96(2):266-70.

Cite this article as: Haider A, Bano I, Sabzposh NA, Arif SH. Role of chromohysteroscopy in detection of endometrial pathology in abnormal uterine bleeding. Int J Reprod Contracept Obstet Gynecol 2019;8:91620. 